that 11 repetitions were observed in this S's protocol. By Formula 1 the expected number of repetitions is $1.17 \mathrm{amd}$ the difference hetween the observed and expected number is 9.83 . Without going further one might conclude that the two Ss evidence almost the same amount of clustering. However. for the first $\mathrm{S}$ the SD (by Formula 2) is 2.40 and for the second $S$ it is .98 . Thus, when these SDs are used to divide the above difference scores, the resulting clustering indexes are 4.17 and 10.03 , respectively. Both valnes are considerably above chance. the second much more than the first. So it is clear that if groups are to be compared for amount of clustcring when the numbers of categories recalled differ from group to group. the standard score method should be used.

Dallett also found differences in number of words recalled with a maximum mean of about 13.5 for the two-category group and a minimum of 10.5 for the eight. From Table 2 the effect of this would be to reduce differences in clustering. although in this case, the difference would be relatively small. It is clear, however, that any time inclependent or task variables effect differentially the number of words recalled, the standard score method should be used, even if the number of categories represented from group to group is the same.

Several indexes of clustering have appeared in the literature
(Cohen. Sakoda. \& Bousticla. 195+1 and it is very possible that some are superior to the standard score index for specilit purposes. However, when the Bouslield \& Bousfield $(1960)$ measure is to be used SD should be taken into account.

RHIERENCLS

BARTON, D. E., \& DAVID, V. N. Nultiple runs. Biometriki, 1957, 44. $168-178^{\circ}$.

BATTIG, W. I.. ALIEN. A. R.. \& JlESLN, A. R. Priority of free recall of newly learned items. Journal of Verbal Learning \& Verbal Behavior. $1965,4,175-179$

BOUSFIELD, A. K., \& BOLSFIELJ). W. A. Medsurement of clusterin: and of sequential constancies in repeated free recall. Psychological Reports, 1966, 19,935-94?.

COHEN, B. H., SAKODA. J. M. \& BOL'SFIFLD. W, A. The statistical analysis of the incidsme of clustering in the recall of randomy arranged associates. Technical Report No, 10, 1954, Iniversity of Connecticut, Contract Nonr-631(00), Office of Naval Research.

DALLFTT, K. M. Number of categories and category information in fre recall. Journal of Experimental Psychology, $1964,68,1-12$.

NOTFS

1. We would like to acknowledge the computational assistance of $\mathrm{Mr}$. Richard Edwards.

2. Present address: Department of Pstchology. Mississippi Stall University, State College, Miss. 39762.

\title{
Recall and anticipation methods in probabilistic associative learning'
}

\section{JAMES F. VOSS, University of Pitsburgh, Pittsburgh, Pa. 15213}

Six $S-R_{1}, S-R_{2}$ modified paired-associates were presented for 60 trials. $A \geq$ by 6 factorial design was employed in which method, recall or anticipation, and the $S-R_{1}, S-R_{2}$ probability-ratio were laried. The $S-R_{1}, S-R_{2}$ ratios were: $1.00-0.00, .90-.10, .80-.20, .70-.30, .60-.40$, and $.50-.50$. The results indicated that the recall procedure yielded superior performance in early acquisition. In later stages, recall vielded results approaching matching. whereas the anticipation procedure yielded overmatching of $R_{1}$ anticipations and undermatching of $R_{2}$ anticipations. The results were interpreted as showing that as probability-ratio varies from $.50-.50$ to $1.00-0.00$, and hence, as the relative difference of response strengths of $S-R_{1}$ and $S-R_{2}$ increases, the selection factor in the anticipation procedure vields a healy weighting of the stronger response, $R_{1}$, and the acquisition of the weaker association, $S-R_{2}$, occurs slowly for the recall paradigm. however, the data suggest that $R_{1}$ is inhibited and $S-R_{2}$ is more readily acquired. The results also were interpreted as showing that one difference in the standard paired-associate recall and anticipation paradigns is that in the recall method. strong error tendencies may be inhibited, but in the anticipation method, strong error tendencies are weighted heavily.

In recent years the traditional paired-associate paradigm has been modified in order to study probabilistic rather than invariant occurrence of responses (e.g. Erdelyi, Watts, \& Voss. 1964; Goss \& Sugarman, 1961: Voss, Thompson, \& Keegan. 1959). The procedure in such experiments has been to pair a stimulus with two (or more) responses, with the stipulation that only one of the responses is presented with the stimulus on any particular trial. Moreover. in the single-stimulus, two-response situation. $S-R_{1}$ and $S-R_{2}$ usually have a fixed probability-ratio, e.g.. .70-.30, with $S-R_{1}$ and $S-R_{2}$ occurrence randomized over trials. In addition. the anticipation method always has been used.

The purpose of the present experiment was to compare the acquisition of $S-R_{1}$. $S-R_{2}$ associations under conditions of recall $(R)$ and anticipation (A) and under probability-ratios varying from $1.00-0.00$ to $.50-.50$. In the paradigm. S-R $\mathrm{R}_{1}$ or $S-R_{2}$ is presented on a particular trial and subsequently upon presentation of the stimulus. $S$ is asked to try to recall the correct response, i.e., $R_{1}$ or $R_{2}$, that was presented with the stimulus on the same trial. Thus, the correct response is the response that most recently was presented with the stimulus. In the $S-R_{1} . S-R_{2}$ anticipation paradigm. the stimulus is presented on a particular trial and $S$ is asked to try to anticipate the response that he thinks will be presented on that particular trial. In this case, the correct response also is designated as the response. $R_{1}$ or $R_{2}$. that occurs on that particular trial. However, with $R_{1}$ and $R_{2}$ randomized. $S$ cannot know whether it is $R_{1}$ or $R_{2}$ that is correct when lie makes his response. The major difference. therefore, in the $S-R_{1}, S-R_{2}$ recall and anticipation paradigms is that in the former. whether $R_{1}$ or $R_{2}$ is correct is designated before $S$ responds, but in the latter $R_{1}$ or $R_{2}$ is designated after he responds. Furthermore, it also should be pointed out that in the recall procedure, $100 \%$ correct responses is possible: in the anticipation procedure, however, with $R_{1}$ and $R_{2}$ randomized, $100 \%$ correct responses is a virtual impossibility.

Because of the previous findings which have indicated overmatching in the $.60-.90$ recall probability conditions, e.g. Voss, Thompson, \& Keegan (1959), and because of the differences in the recall and anticipation paradigms, it was expected that Condition $\mathrm{R}$ would yield more correct responses and performance more closely approximating matching behavior. In addition to these hypotheses, which are somewhat obvious from the comparison of the recall and anticipation paradigms, it was anticipated that the relative difference of the recall and anticipation conditions would increase as the probability-ratio varied from $1.00-0.00$ to $.50-.50$. Such performance was expected on the basis of the possible increase 


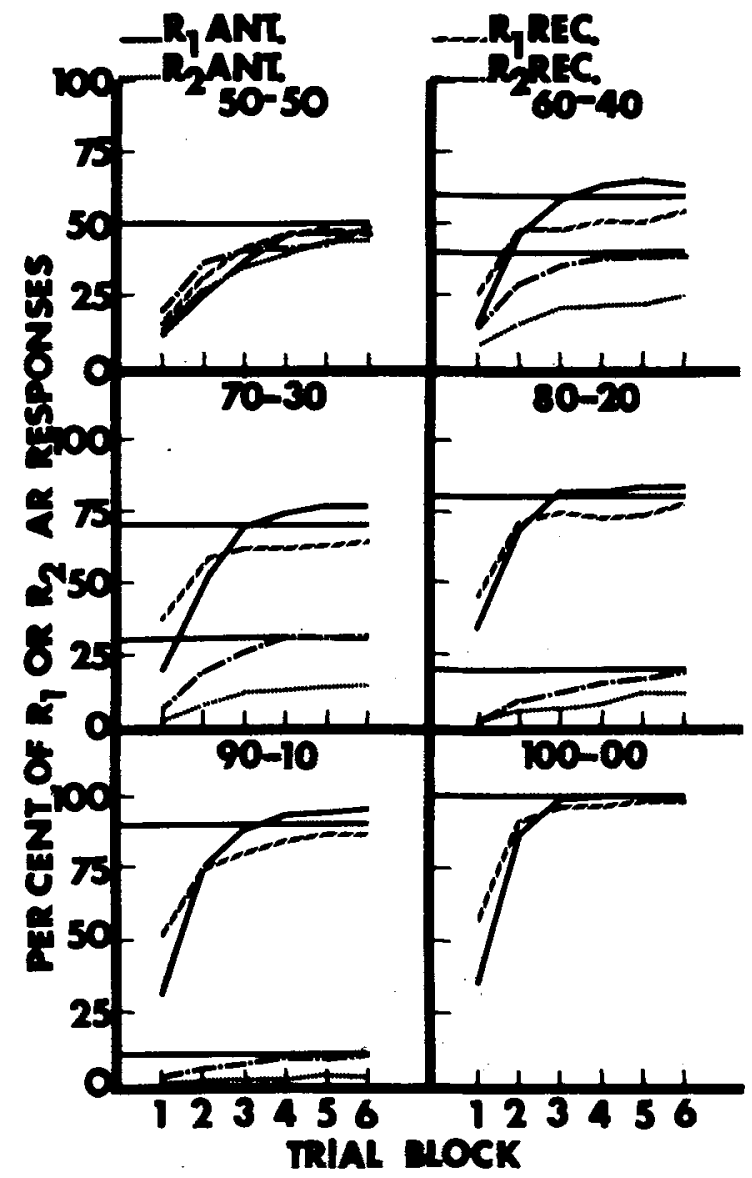

Fig. 1. Per cent $R_{1}$ and $R_{2}$ appropriate responses (AR) as a function of trial block for all probability-ratio conditions.

in guessing and/or response competition in Condition $\mathrm{A}$ as the probability-ratio varied from $1.00-0.00$ to $.50-.50$.

\section{PROCEDURE}

The experiment consisted of a 2 by 6 factorial design with method, anticipation vs recall, and $S-R_{1}, S-R_{2}$ probabilityratio (PR) constituting the two variables. The $P R$ values were: 1.00-0.00, .90-.10,.80-.20,.70-.30,.60-.40, and .50-.50. $\left(\mathbf{R}_{1}\right.$ designates the larger probability within each ratio, and $\mathbf{R}_{2}$ designates the smaller probability. For the purposes of analysis, $R_{1}$ and $R_{2}$ designations were assigned arbitrarily in the $.50-50$ condition.)

In each condition, six modified paired-associates were presented for 60 trials. In Condition $R$, the six pairs were presented on each trial followed by presentation of the same stimulus and the respective response $\left(R_{1}\right.$ or $\left.R_{2}\right)$ designated for that trial. Presentations in both recall and anticipation occurred at a 2-sec rate with a 4-sec intertrial interval.

The six modified paired-associates within each PR condition had the same response probability-ratio. Six different random orders of $R_{1}$ and $R_{2}$ occurrence were employed for the six respective $S-R_{1}, S-R_{2}$ associates within each $P R$ condition. The $R_{1}$ and $R_{2}$ responses were randomized within 10-trial blocks and the random orders of $R_{1}$ and $R_{2}$ occurrence were identical in Conditions $A$ and $R$. In Condition $R$, a different random order was employed in each trial for testing than that used in pairing presentations.

\section{MATERIALS}

Low-frequency words were used (Horton \& Kjeldergaard, 1961). In each of the 12 conditions, the two-response words assigned to $R_{1}$ and $R_{2}$ for a particular $S-R_{1}, S-R_{2}$ combination were counterbalanced for one-half of the Ss.

\section{SUBJECTS}

The Ss were introductory psychology students at the University of Pittsburgh. The N/ condition was 12 and the 144 Ss were assigned to the conditions via a table of random numbers.

\section{RESULTS}

The term "appropriate response" (AR) in Conditions A and $R$ refers to S's statement of either $R_{1}$ or $R_{2}$ to the appropriate stimulus, regardless of whether $R_{1}$ or $R_{2}$. occurred on that particular trial. 2 The term "correct response" (CR) in Conditions $A$ and $R$ refers to S's statement of $\mathbf{R}_{\mathbf{1}}$ or $\mathbf{R}_{2}$ to the appropriate stimulus when that response $\left(R_{1}\right.$ or $\left.R_{2}\right)$ occurred on that particular trial. The term "opposite correct response" (OCR) refers to $S$ 's statement of $R_{1}$ to the appropriate stimulus when $R_{2}$ occurred on that trial or a response of $\mathbf{R}_{2}$ when $R_{1}$ occurred on that trial. Thus, $A R$ frequency $=C R$ frequency + OCR frequency.

$A R$. Figure 1 presents the per cent $\dot{\mathrm{R}}_{1}$ and $\mathrm{R}_{2}$ appropriate responses as a function of trial block for the experimental conditions. The data of the anticipation condition are in general agreement with previous findings (Goss \& Sugarman, 1961; Voss, Thompson, \& Keegan, 1959). The data of Fig. 1 indicate that recall tended to yield more AT responses in earlier trials than Condition $A$, and that Condition $A$ tended to yield more $R_{1}$ and fewer $R_{2} A R$ responses than Condition $R$ in later trials.

Table 1 presents the mean $R_{1}+R_{2}, R_{1}$, and $R_{2} A R$ frequencies for the experimental conditions. An analysis of variance revealed that for the $R_{1}+R_{2}$ data, mean $A R$ frequency was significantly greater for recall than for anticipation $[F(5,132)=7.32, p<.01]$. The $A$ vs $R$ by $P R$ interaction is not significant $[F(5,132)<1.00]$. The significant $A$ vs $R$ by Trial Block (TB) interaction $[F(5,660)=21.76, p<.01]$ indicates that Condition $R$ yielded more $A R$ responses than Condition $A$ in early trials. In addition, AR frequency increased more rapidly over trials as PR varied from $.50-.50$ to $1.00-0.00[F(25,660)=2.79$, $\mathrm{p}<.011$.

Analysis of the $R_{1} A R$ data revealed that $R_{1} A R$ frequency increased as probability-ratios varied from $.50-.50$ to $1.00-0.00$ $[F(5,132)=99.29, p<.01]$, but the $A$ vs $R$ and the PR by $A$ vs $R$ sources of variation are not significant. The analysis also revealed, however, that the Trial Block by $A$ vs $R$ source of variation is significant $[F(5,660)=32.79, p<.01]$; as is the Trial Block by $A$ vs $R$ by $P R$ source of variation $[F(25,660)=2.41, p<.01]$. Inspection of Fig. $I$ indicates that $R_{1} A R$ frequency increased more rapidly in early trials in Condition $R$ than in Condition $A$, especially for PR approaching .50-.50. However, at the second or third trial block, the $A R$ frequency of $R_{1}$ responses in Condition $A$ was greater than in Condition $R$. The lack of a significant difference in total $R_{1}$ anticipations in Conditions $A$ and $R$ is likely due to the relative change in $A$ and $R$ response frequency as a function of trials.

Analysis of the $R_{2} A R$ data revealed that appropriate response frequency increased over the .10 to $.50 \mathrm{PR}$

Table 1

Mean $\mathbf{R}_{1}+\mathbf{R}_{2}, \mathbf{R}_{1}$ and $\mathbf{R}_{2}$ Appropriate Responses (AR) Responses for the Experimental Conditions

\begin{tabular}{cccccrr}
\hline & \multicolumn{2}{c}{$\mathbf{R}_{\mathbf{1}}+\mathbf{R}_{\mathbf{2}}$} & \multicolumn{2}{c}{$\mathbf{R}_{\mathbf{1}}$} & \multicolumn{2}{c}{$\mathbf{R}_{\mathbf{2}}$} \\
$\mathbf{P R}$ & $\mathbf{A}$ & $\mathbf{R}$ & $\mathbf{A}$ & $\mathbf{R}$ & \multicolumn{1}{c}{$\mathbf{A}$} & \multicolumn{1}{c}{$\mathbf{R}$} \\
\hline $1.00-0.00$ & 312.4 & 322.1 & - & - & - & - \\
$.90-.10$ & 291.3 & 303.3 & 286.3 & 278.6 & 5.0 & 24.7 \\
$.80-.20$ & 284.9 & 291.5 & 259.4 & 248.3 & 25.5 & 43.2 \\
$.70-.30$ & 257.6 & 293.3 & 222.1 & 208.8 & 35.5 & 84.5 \\
$.60-.40$ & 253.3 & 280.3 & 186.3 & 165.5 & 67.1 & 114.8 \\
$.50-.50$ & 248.9 & 274.9 & 127.8 & 136.7 & 121.1 & 138.3 \\
\hline
\end{tabular}


Table 2

Mean $\mathbf{R}_{1}+\mathbf{R}_{2}, \mathbf{R}_{1}$, and $\mathbf{R}_{2}$ Correct Responses (CR) for the Experimental Conditions

\begin{tabular}{rrrrrrr}
$\mathbf{P R}$ & \multicolumn{1}{c}{$\mathbf{A}$} & \multicolumn{1}{c}{$\mathbf{R}_{\mathbf{1}}+\mathbf{R}_{\mathbf{2}}$} & \multicolumn{2}{c}{$\mathbf{R}_{\mathbf{1}}$} & \multicolumn{2}{c}{$\mathbf{R}_{\mathbf{2}}$} \\
\hline $1.00-0.00$ & 312.4 & 322.1 & - & - & - & - \\
$.90-.10$ & 256.1 & 297.1 & 255.9 & 273.3 & 0.2 & 23.8 \\
$.80-.20$ & 208.8 & 277.2 & 204.7 & 236.1 & 4.1 & 41.1 \\
$.70-.30$ & 161.3 & 251.2 & 152.8 & 187.4 & 8.6 & 63.8 \\
$.60-.40$ & 135.7 & 230.7 & 109.9 & 143.7 & 25.8 & 87.0 \\
$.50-.50$ & 121.3 & 219.0 & 62.8 & 109.2 & 58.5 & 109.8 \\
\hline
\end{tabular}

$[F(4,110)=88.39, p<.01]$, that Condition $R$ yielded a greater $R_{2} \quad A R$ frequency than Condition $A$ $[F(1,110)=48.57, p<.01]$, and that the difference in anticipation and recall conditions increased as $R_{2} P R$ varied from .50 to $.10[F(4.110)=2.90, p<.05\}$. In addition, the Trial Block by $\mathrm{PR}$ source of variation is significant $[F(20,550)=10.16, p<.01]$, as is the interaction of Trial Block by $A$ vs $R[F(5.550)=6.01, p<.01]$, and the Trial Block by $A$ vs $R$ by $P R$ interaction $[F(20,550)=1.94$. $p<.01]$. These findings indicate that $R_{2} A R$ frequency was greater for Condition $R$ than Condition $A$, that this difference increased as $R_{2}$ probability varied from .10 to .50 , and that such an increase became greater over trials.

$C R$. Table 2 presents mean correct $\mathrm{R}_{1}+\mathrm{R}_{2}, \mathrm{R}_{1}$, and $\mathrm{R}_{2}$ responses for the experimental conditions. For all three measures, recall, as expected, yielded more correct responses than anticipation: $R_{1}+R_{2}, F(1.132)=147.92, p<.01$. There also were more $R_{1}+R_{2}$ correct responses as $P R$ varied from $.50-.50$ to $1.00-0.00[F(5.132)=71.51, p<.01]$, and the increase over $P R$ was greater in Condition $R$ than in Condition A $[F(5.132)=6.84, p<.01]$.

With respect to the $R_{1}$ and $R_{2} C R$ data, the per cent correct response at each probability condition was computed by each $S$ because opportunities for a correct response increased systematically from .50 to 1.00 for $R_{1}$ data and increased systematically from .10 to .50 for the $R_{2}$ data. Analysis of the $R_{1}$ arc sin transformed per cent correct response data revealed that per cent correct responses in anticipation was greater than in recall $[F(1.132)=38.22, p<01]$ : per cent correct responses increased as the probability ratio varied from .50 to $1.00[F(5,132)=58.08 . p<.01]$, and that the difference in per cent correct response in Conditions $R$ and $A$ increased as $P R$ varied from 1.00 to .50 . The arc sin transformed per cent correct response $R_{2}$ analysis also revealed a greater $R_{2}$ correct response in Condition $R$ than in Condition $A$ $[F(1.110)=433.19, p<.01]$. an increase in per cent correct responses as $R_{2}$ varied from .10 to $.50(\mathrm{~F}(4.110)=8.72$, $\mathrm{p}<.011$, and a relatively greater difference in Conditions $R$ and $A$ occurred as $R_{2}$ varied from .50 to .10 $[F(4.110)=13.29 . p<.01]$.

$O C R$. Figure 2 presents per cent opposite correct responses for the experimental conditions. The percentage data were obtained by dividing the number of presentations of a particular response, e.g. 42 for an $R_{1}$ response in the .70-.30 condition, into $R_{2}$ occurrences during the $42 R_{1}$ presentations. Thus, these data take into account the different number of presentations of $R_{1}$ and $R_{2}$ in the respective $P R$ conditions. An analysis of variance performed on the $R_{1}$ OCR data revealed that a greater percentage of $R_{1}$ responses occurred on $R_{2}$ presentations as $P R$ varied from .50 to .90 $[F(4,110)=16.69, p<.01]$, that Condition $A$ had a significantly greater percentage of such responses than Condition $\mathbf{R}$ $[F(1,110)=533.99, p<.61]$, and the difference in anticipation and recall decreased as the probability ratio varied from .90 to $.50[F(4.110)=18.89, p<.01]$. Further analysis of the individual curves indicated that in Condition $A$, pers cent $R_{1}$ responses on $R_{2}$ presentations decreased linearly over PR $[F(1,55)=137.21$. $p<.01]$. For the recall condition, however, there is no significant curve component.

With respect to $R_{2}$ responses on $R_{1}$ presentations, there was significantly greater per cent opposite correct response percentage in Condition $A$ than in Condition $R$ $[F(1,110)=39.12, p<.01]$, a greater per cent of $O C R$ responses as $P R$ varied from .10 to $.50(F(4.110)=74.22$. $p<.01]$, and the difference in Conditions $R$ and $A$ decreased as $P R$ varied from .50 to $.10[F(4,110)=4.71, p<.01]$. Further analysis revealed that per cent $R_{2}$ occurrences when $R_{1}$ was presented increased linearly in Condition $A$ as $P R$ varied from .10 to $.50 \quad[F(1,55)=183.48, p<.01]$. In Condition $R$, the same effect was found $[F(1.55)=116.51$, $\mathrm{p}<.01]$.

The Condition A OCR findings thus indicate that as PR varies from $.90-10$ to $.50-.50$ per cent. $R_{1}$ response frequency decreases when $R_{2}$ is presented and per cent $R_{2}$ response frequency increases when $R_{1}$ is presented. For Condition $R$ however, only per cent $R_{2}$ OCR increased over PR.

\section{DISCUSSION}

The $A R$ and $C R$ data indicate that performance in Condition $\mathrm{R}$ was characterized by a gradual increase in correct $R_{1}$ and $R_{2}$ recalls such that $S$ s apparently were approaching the asymptotic level of matching which, in the recall method. would yield $100 \%$ correct responses. This finding, at first glance. suggests that in Condition $R$, the $S-R_{1}$ and $S-R_{2}$ associations are acquired independently. The OCR data however, are in opposition to this view since these data indicate that $R_{1}$ interfered with $R_{2}$ acquisition and $R_{2}$ interfered with $R_{1}$

Of particular interest is the finding that although there was a greater percentage of $R_{1}$ recalls on $R_{2}$ trials than the reverse the $R_{1}$ recalls on $R_{2}$ trials did not vary significantly with probability ratio. The likely reason for this result is suggested by an experiment by Bloch \& Voss (1967) who found, in the $S-R_{1}$. S-R $R_{2} .50-.50$ recall situation. that strong $S-R_{1}$ normative associates provided less interference in $R_{2}$ acquisition than weak associates. The hypothesis was advanced that strong $R_{1}$ associates may be inhibited during $R_{2}$ acquisition. In the present experiment. it may be suggested that the reason the per cent $R_{1} O C R$ responses did not increase over $P R$ from .50 to .90 is that the $R_{1}$ responses could be inhibited during $R_{2}$ acquisition. In other words, as $S-R_{1}$ strength increased. it became easier for $S$ to inhibit $R_{1}$ in the learning of $S-R_{2}$. On the other hand. the strength of $S-R_{2}$, never reaching the level

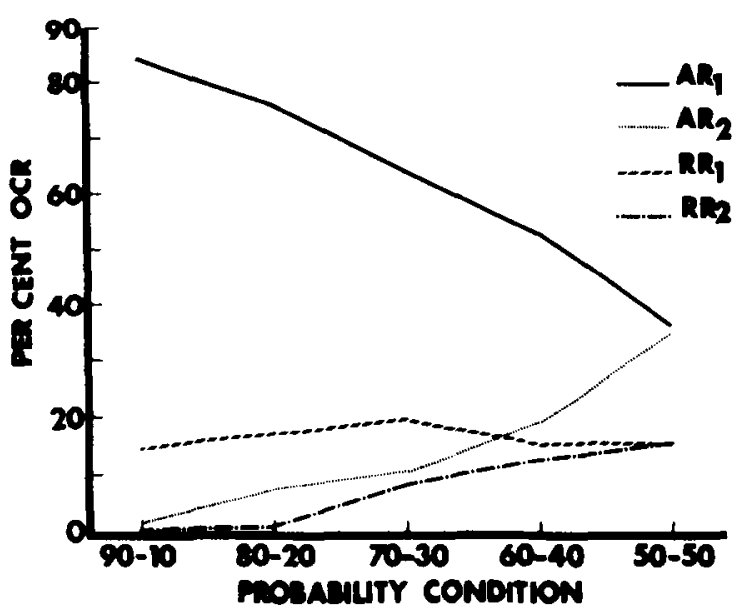

Fig. 2. Per cent opposite correct responses (OCR) as a function of probability condition. 
of $S-R_{1}$, except at the PR of .50-.50, did produce increasing interference over the PR of .10 to .50 . This finding also may be related to the Bloch \& Voss (1967) results in that moderately strong $S-R_{1}$ associates interfered more in the acquisition of $S-R_{2}$ associates than weak associates. In summary, the Condition $R$ results suggest that as $S-R_{2}$ strength increases, over .10 to .50 , and becomes more equated to $S-R_{1}$ strength, $S-R_{2}$ produces increasing interference; as $S-R_{1}$ strength increases over .50 to .90 , the interference produced is constant. with the explanation of fered that the Ss inhibit the stronger $R_{1}$ responses.

The $A R$ and $C R$ data indicate that performance in Condition $A$ was characterized by a gradual increase in $R_{1}$ and $R_{2}$ anticipations with the finding of overmatching of $R_{1}$ anticipations and undermatching of $R_{2}$. It may be assumed that the random nature of the $S-R_{1}, S-R_{2}$ sequence produced fewer correct responses than in the recall condition.

Of particular interest is the finding that $R_{2}$ occurrences on $R_{1}$ trials and $R_{1}$ occurrences on $R_{2}$ trials both vary linearly over probability ratios. The most reasonable explanations for these data is in terms of either response competition or guessing behavior in the Condition A paradigm. Specifically, it may be assumed that as $P R$ is varied from $.50-.50$ to $.90-.10$, the relative differences of $S-R_{1}$ and $S-R_{2}$ strength increases. With respect to performance, as $\mathrm{PR}$ varies from .50-.50 to $90-10, R_{1}$ response frequency relative to $R_{2}$ increases. Thus, as the relative difference in the strength of the $S-R_{1}$ and $S-R_{2}$ associates increases, the selection factor in the $A$ paradigm produces an increasing weighting of $R_{1}$ responses. This finding supports previous results (Erdelyi, Watts, \& Voss, 1964). With respect to guessing, the relative increase of performance of $R_{1}$ compared to $R_{2}$ could be attributed to a guessing factor compared to $R_{2}$ could be attributed to a guessing factor involving a more likely chance of being correct with an $R_{1}$ rather than an $R_{2}$ response.

Comparison of the anticipation condition and recall condition $S-R_{1}$. S- $R_{2}$ paradigms therefore leads to the following tentative conclusion: As the relative differences of $S-R_{1}, S-R_{2}$ probability tatios increases from $.50-.50$ to $.90-.10$, the role of the $R_{1}$ responses varies as a function of procedure; in Condition R. strong responses tend to be inhibited in the acquisition of $S-R_{2}$ associates, while in Condition $A$, strong responses tend to be weighted and produce slower acquisition of $\mathrm{S}-\mathrm{R}_{2}$ associates.

The present findings are relevant to experiments designed to compare the standard paired-associate recall and ancitipation tasks. Evidence for the superiority of the recall procedure has been provided (e.g., Battig \& Brackett, 1961) whereas other experiments (e.g.. Battig \& Brackett, 1963: Cofer, Diamond, Olsen. Stein.\& Walker, 1967) have failed to yjeld consistent re- sults. The present findings suggest that a major factor involved in the comparison of the recall and anticipation methods is the degree of response competition within a list. Specifically, it would be expected that the role of error tendencies within a list interacts with method: when incorrect error tendencies are relatively large, the incorrect responses may be more easily inhibited in the recall procedure and weighted more heavily in the anticipation method. Little direct evidence, however, is available to either support or not support this hypothesis, although recent work on the role of interpair interference in paired-associate acquisition (e.g., Brown, 1964; Voss, 1967) provides a method to test such a hypothesis.

\section{REFERENCES}

BATTIG, W. F., \& BRACKETT, H. R. Comparison of anticipation and recall methods in paired-associate learning. Psychological Reports, $1961,9,59-65$.

BATTIG, W. F., \& BRACKETT, H. R. Transfer from verbaldiscrimination to paired-associate learning: II. Effects of intralist similarity, method, and percentage occurrence of response members. Joumal of Experimental Psychology, 1963,65, 507-514.

BLOCH, R., \& VOSS, J.F. Acquisition of paired associates as a function of associative strength of competing responses. Journal of Experimental Psychology, 1967, 74, 14-19.

BROWN, S. C. Interpair interference as a function of level of practice in paired-associate learning. Journal of Experimental Psychology, 1964, 67, 316-323.

COFER, C. N., DIAMOND, F, OLSEN, R. A., STEIN, J. S., \& WALKER, H. Comparison of anticipation and recall methods in paired-associate learning Journal of Experimental Psychology, 1967, $75,545-558$.

ERDELYI, M., WATTS, B., \& VOSS, J. F. Effect of probability of competing responses in probabilistic verbal acquisition. Journal of Experimental Psychology, 1964, 68, 323-329.

GOSS, A. E, \& SUGARMAN, M. E. Paired-associate learning with varying relative percentages of occurrence of alternative response members. Journal of Experimental Psychology, 1961, 62, 24-34.

HORTON, D. L., \& KJELDERGAARD, P. M. An experimental analysis of associative factors in mediated generalizations. Psychological Monographs: General \& Applied, 1961, 75, (11, Whole No. 515).

vOSS, J. F. Intralist interference in associative learning. Journal of Verbal Learning \& Verbal Behavior, 1967, 7, 773-779.

vOSS, J. F., THOMPSON, C. P., \& KEEGAN, J. H. Acquisition of probabilistic paired associates as a function of S-R $R_{1}, S-R_{2}$ probability. Journal of Experimental Psychology, 1959, 58, 390-399.

$$
\text { NOTES }
$$

1. The research reported in this paper was supported by the National Institute of Child Growth and Human Development (00957-03). The author wishes to thank Barbara Watts and Jay Jordan for assistance in the collection and analysis of the data and Dr. W. Battig for his comments on the manuscript.

2. The term, "appropriate response," is used in the same way that $A_{1}$ and $A_{2}$ are used in probability learning experiments to designate anticipations of $R_{1}$ and $R_{2}$, respectively. "Appropriate-response" rather than "anticipation" is employed in the present experiment in order to avoid the confusion possible in using the word, "anticipation," to refer to one of the procedures and to a type of response. 